\title{
The therapeutic effects of bone marrow mesenchymal stem cells after optic nerve damage in the adult rat
}

This article was published in the following Dove Press journal:

Clinical Interventions in Aging

16 February 2015

Number of times this article has been viewed

\author{
HaiBo Tan ${ }^{1, *}$ \\ Xin Kang ${ }^{2, *}$ \\ ShiHeng Lu' \\ Lin Liu' \\ 'Department of Ophthalmology, Renji \\ Hospital, The School of Medicine, \\ Shanghai JiaoTong University, \\ Shanghai, People's Republic of China; \\ 2Department of Clinical Pharmacology, \\ Changhai Hospital Affiliated Second \\ Military Medical University, Shanghai, \\ People's Republic of China \\ *These authors contributed equally \\ to this work
}

\begin{abstract}
Optic nerve trauma is a common occurrence that results in irreversible blindness. Currently, no effective strategies are known to prevent optic nerve degeneration. We assessed the therapeutic effects of bone marrow mesenchymal stem cells (BMSCs) after optic nerve crush in the adult rat. Our results showed that BMSCs significantly promoted the regeneration of injured axons compared with phosphate buffered saline alone. Therefore, BMSC transplantation may be effective for the treatment of central nervous system disorders.
\end{abstract}

Keywords: bone marrow mesenchymal stem cell, axonal regeneration, rat optic nerve crush, nervous system disorders

\section{Introduction}

Optic nerve damage is an important cause of irreversible vision loss, which results from retinal ganglion cell (RGC) death and subsequent axonal degeneration. ${ }^{1,2}$ It is well known that in the central nervous system, the failure of axonal regeneration is due not only to the limited intrinsic growth capacity of adult neurons but also to the growth inhibitory environment. ${ }^{3}$ Therefore, therapies should focus on the protection of injured neurons and on the promotion of axonal regeneration. ${ }^{4-8}$ Cell transplantation therapy has been proven effective in this regard. ${ }^{9}$

Bone marrow mesenchymal stem cells (BMSCs) have both amplification and transplantation abilities. ${ }^{10,11}$ Furthermore, BMSCs have been shown to exert a neuroprotective effect on RGCs. ${ }^{12}$ However, little is known about the effects of BMSCs on axonal regeneration of the injured optic nerve. Here, we explored the axonal regeneration-promoting effect of BMSCs after optic nerve crush (ONC).

\section{Materials and methods ONC and intravitreal injection}

This experiment was approved by the Ethics Committee of Animal Research of RenJi Hospital, The School of Medicine, Shanghai JiaoTong University. Eighty adult male Sprague Dawley rats (weight: 200-250 g) were provided by the Shanghai Institute of Traumatology and Orthopedics and were housed in a temperature- and humiditycontrolled room with a 12:12 hour light:dark cycle.

Surgical procedures were performed as described in our previous study. ${ }^{13}$ The right optic nerve was crushed $1 \mathrm{~mm}$ behind the posterior eye pole for 9 seconds for all experimental groups. But the optic nerve was not crushed in the sham-surgery group. BMSCs were purchased from Shanghai Shengong Biotechnology (Shanghai Shengong Biotechnology, Shanghai, People's Republic of China). A total of $3 \mu \mathrm{L}$ of
Department of Ophthalmology, Renji Hospital, The School of Medicine, Shanghai JiaoTong University, Shanghai 200025, People's Republic of China Tel +86 I36 2I I6 0754

Email thbbobo@।26.com 
phosphate buffered saline (PBS) or BMSCs (low number of transplantation cells: $3 \times 10^{4}$; high number of transplantation cells: $1 \times 10^{5}$ ) were intravitreally injected immediately, and 3,6 , and 9 days after ONC. Rats in the sham surgery group did not receive an intravitreal injection. Rats with lens injury were excluded. These rats were divided into four groups: the sham surgery group, PBS group, low BMSC group, and high BMSC group. Twenty rats in each experimental group were included in the final evaluation.

\section{Processing of optic nerve samples}

The procedures were performed as described in our previous study. ${ }^{13}$ The rats were sacrificed on day 15 after ONC and were transcardially perfused with $4 \%$ paraformaldehyde. The optic nerves were dissected and immediately fixed in $4 \%$ PFA in paraformaldehyde for 1 hour. Then, the tissue was embedded, frozen, and cut. Longitudinal sections (16 $\mu \mathrm{m}$ thickness) of the optic nerve were obtained and were placed on gelatin-coated glass slides for subsequent use.

\section{Axon count}

With respect to the axon count, the optic nerve sections were dehumidified at $37^{\circ} \mathrm{C}$ for 1 hour, and nonspecific binding was blocked by the application of $10 \%$ normal goat serum. To stain the regenerating fibers, an anti-GAP43 antibody (mouse anti-rat GAP-43 monoclonal antibody, 1:100, sc-17790; Santa Cruz Biotechnology Inc., Dallas, TX, USA) was applied and the sections were incubated at $4^{\circ} \mathrm{C}$ overnight. After two washes with PBS, the secondary antibody (Cy-3-labeled anti-mouse fluorescein isothiocyanate antibody, 1:100; Thermo Fisher Scientific, Waltham, MA, USA) was applied and the sections were incubated for 45 minutes at room temperature. GAP-43-positive axons were quantified to assess the regeneration of the axons of RGCs as previously described. ${ }^{13}$

\section{Western blot analysis}

Western blot analysis was used to determine the expression levels of GAP-43 after ONC. The methods of Western blot analysis were described in our previous study. ${ }^{13}$

\section{Statistical analyses}

The data analyses were performed using SPSS version 19.0 software (IBM Corporation, Armonk, NY, USA), and the data are expressed as the mean \pm standard deviation. The differences between the groups were determined by one-way analysis of variance or by independent samples Student's $t$-test. A $P<0.05$ was considered statistically significant.

\section{Results \\ BMSC promotes the axonal regeneration of rat RGCs following ONC}

Our results indicated very few axons beyond the crush site in the PBS-treated group. However, a large number of GAP-43-positive axons passed through the crush site in the BMSC-treated group (low or high transplantation group) on day 15 after ONC (Figure 1). Rats in the sham surgery group showed no significant staining (Figure 1). The data
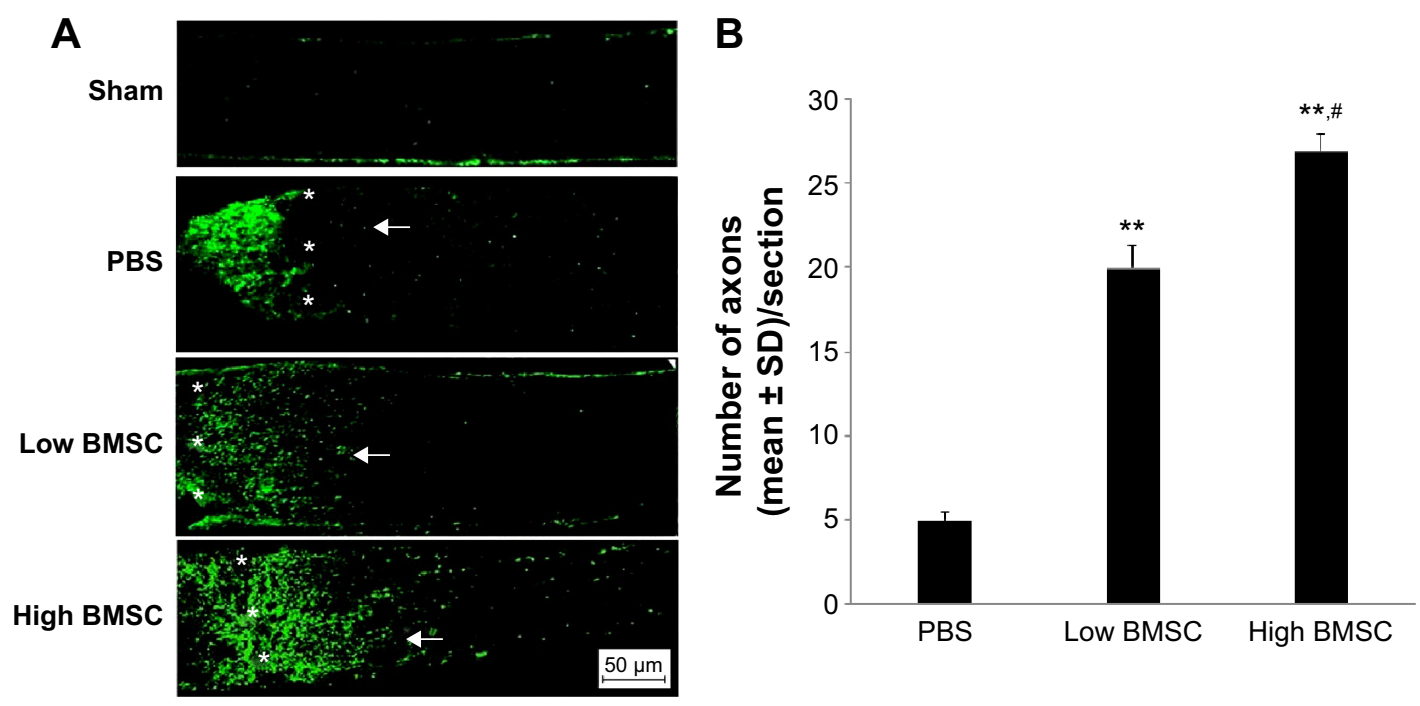

Figure I Representative photomicrographs of regenerating axons in all groups after ONC.

Notes: The numbers of axons/section are presented as the mean \pm SD $(n=10)$. (A) asterisk, the crush site; arrow heads, GAP-43 immunofluorescence; scale bar, $50 \mu m$. (B) ${ }^{* * P}<0.00$ I compared with the PBS group; ${ }^{\# P}<0.05$ compared with the low BMSC group.

Abbreviations: ONC, optic nerve crush; SD, standard deviation; BMSC, bone marrow mesenchymal stem cell; PBS, phosphate buffered saline. 

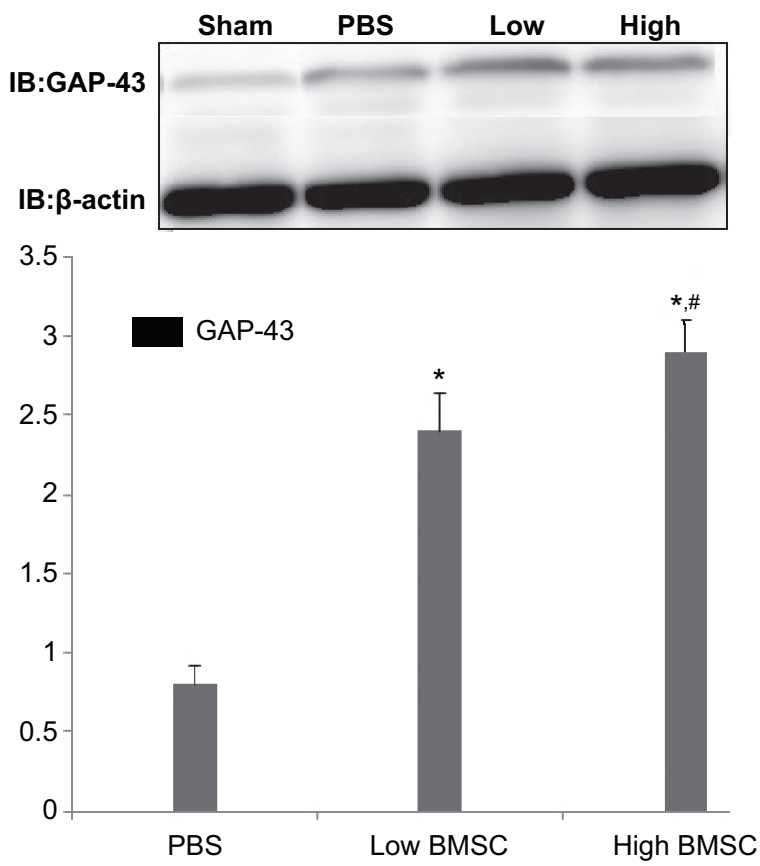

Figure 2 Western blot analysis of GAP-43 expression.

Notes: $* P<0.001$, compared with the PBS group; ${ }^{*} P<0.05$, compared with the low BMSC group.

Abbreviations: BMSC, bone marrow mesenchymal stem cell; IB, immunoblotting; PBS, phosphate buffered saline.

analysis suggested that more axons were present in the two BMSC groups (low and high) than in the PBS group $(P<0.001)$. Moreover, the high BMSC transplantation group was associated with higher numbers of regenerative axons in comparison with the low BMSC transplantation group.

\section{BMSCs increase GAP-43 expression}

According to Western blot analysis, GAP-43 expression was weak in the sham surgery group, but GAP-43 expression was differentially increased in the PBS and BMSC (low and high) groups $(P<0.001$; Figure 2$)$. Moreover, GAP-43 expression in the low and high BMSC transplantation groups was higher than that in the PBS group (Figure 2). In addition, the group with a high number of transplanted cells demonstrated increased axonal regeneration compared with the group with a low number of transplanted cells $(P>0.05$; Figure 2$)$.

\section{Discussion}

The present study explored the effect of transplanted BMSCs on axonal-regeneration after the ONC. GAP-43 expression in rats in the PBS and BMSC groups was significantly increased compared with rats in the sham surgery group, but the GAP-43 protein levels were higher in both BMSC groups than in the PBS group. This result suggested that BMSCs promoted axonal regeneration of the injured optic nerve.
The mechanism of how BMSC therapy might be effective for axonal regeneration after ONC has not yet been elucidated. The function of transplanted BMSCs may involve the regulation of immune system-related inflammation in the injured tissues. In experimental autoimmune encephalomyelitis, Semon et al demonstrated a significant reduction in inflammatory infiltrates by BMSCs. ${ }^{14}$ Furthermore, BMSCconditioned medium significantly decreased cell proliferation and the secretion of pro-inflammatory factors through activated microglia. ${ }^{15} \mathrm{Li}$ et al also indicated that BMSCs enhanced axonal remodeling in a model of experimental stroke. ${ }^{16}$ On the contrary, BMSCs promoted axonal regeneration via the increase in the production of trophic factors. ${ }^{17} \mathrm{In}$ addition, no significant adverse effects were observed in the two BMSC groups.

In summary, our study demonstrates that BMSCs may be a potential therapeutic option for disease of the central nervous system.

\section{Disclosure}

The authors report no conflicts of interest in this work.

\section{References}

1. Tang Z, Zhang S, Lee C, et al. An optic nerve crush injury murine model to study retinal ganglion cell survival. J Vis Exp. 2011;(50):2685.

2. Yoles E, Schwartz M. Degeneration of spared axons following partial white matter lesion: implications for optic nerve neuropathies. Exp Neurol. 1998;153(1):1-7.

3. Planchamp V, Bermel C, Tönges L, et al. BAG1 promotes axonal outgrowth and regeneration in vivo via Raf-1 and reduction of ROCK activity. Brain. 2008;131(Pt 10):2606-2619.

4. Levin LA. Axonal loss and neuroprotection in optic neuropathies. Can J Ophthalmol. 2007;42(3):403-408.

5. Zalish M, Lavie V, Duvdevani R, Yoles E, Schwartz M. Gangliosides attenuate axonal loss after optic nerve injury. Retina. 1993;13(2): 145-147.

6. Fitzgerald M, Payne SC, Bartlett CA, Evill L, Harvey AR, Dunlop SA Secondary retinal ganglion cell death and the neuroprotective effects of the calcium channel blocker lomerizine. Invest Ophthalmol Vis Sci. 2009;50(11):5456-5462.

7. Thaler S, Fiedorowicz M, Rejdak R, et al. Neuroprotective effects of tempol on retinal ganglion cells in a partial optic nerve crush rat model with and without iron load. Exp Eye Res. 2010;90(2):254-260.

8. Tsai RK, Chang CH, Sheu MM, Huang ZL. Anti-apoptotic effects of human granulocyte colony-stimulating factor (G-CSF) on retinal ganglion cells after optic nerve crush are PI3K/AKT-dependent. Exp Eye Res. 2010;90(5):537-545.

9. Hou S, Nicholson L, van Niekerk E, Motsch M, Blesch A. Dependence of regenerated sensory axons on continuous neurotrophin-3 delivery. J Neurosci. 2012;32(38):13206-13220.

10. Johnson TV, Martin KR. Cell transplantation approaches to retinal ganglion cell neuroprotection in glaucoma. Curr Opin Pharmacol. 2013; 13(1):78-82.

11. Bouhenni RA, Dunmire J, Sewell A, Edward DP. Animal models of glaucoma. J Biomed Biotechnol. 2012;2012:692609.

12. Hu Y, Tan HB, Wang XM, Rong H, Cui HP, Cui H. Bone marrow mesenchymal stem cells protect against retinal ganglion cell loss in aged rats with glaucoma. Clin Interv Aging. 2013;8:1467-1470. 
13. Tan H, Zhong Y, Shen X, Cheng Y, Jiao Q, Deng L. Erythropoietin promotes axonal regeneration after optic nerve crush in vivo by inhibition of RhoA/ROCK signaling pathway. Neuropharmacology. 2012;63(6):1182-1190.

14. Semon JA, Maness C, Zhang X, et al. Comparison of human adult stem cells from adipose tissue and bone marrow in the treatment of experimental autoimmune encephalomyelitis. Stem Cell Res Ther. 2014; $5(1): 2$.

15. Yan K, Zhang R, Sun C, et al. Bone marrow-derived mesenchymal stem cells maintain the resting phenotype of microglia and inhibit microglial activation. PLoS One. 2013;8(12):e84116.
16. Li Y, McIntosh K, Chen J, et al. Allogeneic bone marrow stromal cells promoteglial-axonal remodeling without immunologic sensitization after stroke in rats. Exp Neurol. 2006;198(2):313-325.

17. Novikova LN, Brohlin M, Kingham PJ, Novikov LN, Wiberg M. Neuroprotective and growth-promoting effects of bone marrow stromal cells after cervical spinal cord injury in adult rats. Cytotherapy. 2011;13(7):873-887.

\section{Publish your work in this journal}

Clinical Interventions in Aging is an international, peer-reviewed journal focusing on evidence-based reports on the value or lack thereof of treatments intended to prevent or delay the onset of maladaptive correlates of aging in human beings. This journal is indexed on PubMed Central, MedLine,
CAS, Scopus and the Elsevier Bibliographic databases. The manuscript management system is completely online and includes a very quick and fair peer-review system, which is all easy to use. Visit http://www.dovepress. com/testimonials.php to read real quotes from published authors. 